\title{
LOS LÍMITES DE LA ALTERIDAD: EL OTRO DESDE LA ENERGÉTICA SOCIAL
}

\author{
The Limits of Otherness: the Other from the Social Energetic Perspective
}

\author{
Alain G. Jullian-Montañez
}

\begin{abstract}
Resumen: La alteridad ha sido abordada desde varias perspectivas, como la educación, el lenguaje, la psicopedagogía, la psicología, la sociología, la antropología o la filosofía, por lo que se ha considerado necesario efectuar una recapitulación de los avances en el tema. Al llevar a cabo esa revisión, se hizo evidente que ninguna de las perspectivas actuales aporta una base material al "problema del otro". Debido a eso, en este artículo se busca, mediante la perspectiva de la energética social, rastrear dicha base material de la alteridad. Esto no responde a una mera curiosidad científica, ya que encontrar dicha base permite replantear las posibles formas de interactuar con el otro.
\end{abstract}

Palabras clave: alteridad, energética social, antropología, estructura disipativa, termodinámica, otro.

Abstract: Otherness has been taken up from many perspectives as Education, Language, Psychopedagogy, Psychology, Sociology, Anthropology and Philosophy. That's why it is necessary to make a recapitulation of the progress about this topic. When performing this review, it became evident that none of the current perspectives provide a solid base to "the Other's problem". Due to this, this article aims to track that material base of Otherness, by the social energetic perspective. This is not simple scientific curiosity, since finding that base allows restating the possible ways to interact with the other.

Keywords: alterity, social energy, anthropology, dissipative structure, thermodynamics, other.

Alain G. Jullian Montañez, doctor en medioambiente y desarrollo por la Universidad Autónoma de Baja California, México. Actualmente en estancia posdoctoral en el Centro de Investigaciones Interdisciplinarias para el Desarrollo Regional de la Universidad Autónoma de Tlaxcala, México. Temas de especialización: ecología, manejo de ecosistemas, alteridad, zoología. Correo electrónico: al304455@yahoo.com.mx.
Enviado a dictamen: 07 de mayo de 2014.

Aprobación: 17 de octubre de 2014.

Revisiones: 1 . 


\section{Introducción}

$\mathrm{D}$ urante el año 2009, González-Silva publicó dos artículos en los que revisó, de manera general, los avances hasta aquel año en la investigación sobre alteridad. Dichos artículos llevan por título "Alteridad y su itinerario desde las perspectivas multidisciplinares" e "Itinerario de alteridad. Una reconstrucción para nuevas aproximaciones" (González-Silva, 2009a y 2009b). En ellos el autor presentó una cronología muy interesante de cómo se había desarrollado el tema de la alteridad en los últimos años. Así, aunque no consideraba a autores como Ricoeur, Skliar, Todorov, Baudrillard y Parrilla-Rubio, destacó las principales perspectivas epistémicas desde las cuales se había abordado la alteridad; es decir, educación, lenguaje, psicopedagogía, psicología, sociología, antropología y filosofía (Baudrillard, 1997; González-Silva, 2009a; Parrilla-Rubio, 2007; Ricoeur, 1996; Skliar, 2002; Todorov, 2001).

Debido a lo anterior, no parece extraño que el segundo artículo publicado aquel año por Freddy González-Silva concluya con el siguiente párrafo:

El análisis precedente permite reorientar las futuras aproximaciones al término. En este sentido, se crean caminosparaeldesarrollode teorías que denexplicaciones máscongruentesalainteracciónhumana.Cabepreguntarse, iserá la alteridad el eslabón perdido que permita el reencuentroverdaderodelos habitantesen estaaldeaglobal? En todo caso, se requiere un acercamiento definitivo a la experiencia de otredad si realmente se desea un mundo mejor (González-Silva, 2009b: 637).

En efecto, se pueden reorientar las aproximaciones a la alteridad partiendo de estas investigaciones por dos razones. En primer lugar, se evidencia la magnitud del fenómeno del otro; es decir, pareciera que al tener tantos matices se complica un abordaje único que dé cuenta de toda la variedad que posee. En segundo lugar, el enlistar las principales perspectivas desde las que se ha abordado la alteridad permite situar aquellos aspectos que no han sido empleados, lo que hace posible señalar las áreas de oportunidad para plantear futuras investigaciones.

De este segundo aspecto se deriva una constante en las investigaciones sobre la alteridad: no se explora su sustento material. Esto resulta notable ya que, pese a la riqueza y variedad de perspectivas, ninguna de ellas plantea la posibilidad de asignar un sustento tangible, en tanto límite físico, al fenómeno de la alteridad. Esto no es un asunto menor ya que reduce la interpretación que se hace de dicho fenómeno a sus elementos culturales e intangibles, aunque el otro necesariamente tiene un componente físico. ${ }^{1}$

¿Qué ventajas tiene indagar sobre el fundamento físico o material de la alteridad? Permite abarcar toda la gama del fenómeno del otro entendiendo sus límites tanto generales como individuales y, en última instancia, ayuda a proponer rutas de convivencia no sólo desde lo intangible, sino desde el mundo físico. ${ }^{2}$

Ahora bien, ¿cómo buscar el fundamento material del otro? En esta investigación se propone la utilización de las ideas de la corriente antropológica denominada "energética social" porque esta forma de abordaje ofrece facilidades para concatenar otras áreas como la biología o la física a la explicación social.

\section{El enfoque de la energética social}

La energética social es una escuela o corriente de la antropología social que se inició a mediados del siglo XX. Esta escuela se centra en entender la sociedad tomando como base la energía, y algunos de sus exponentes más destacados son Leslie White, Richard N. Adams y Leonardo Tyrtania.

El iniciador de la energética social fue Leslie White, quien sostuvo que todo en el universo puede ser descrito en términos de la energía (White, 1943). Sin embargo, en su obra puso el acento en el desarrollo tecnológico, en la satisfacción de necesidades y en la producción de bienes y servicios, por lo que su perspectiva resultó limitada. Además, la física en los tiempos de White todavía no había desarrollado las teorías sobre los sistemas termodinámicamente abiertos, lo cual dificultaba aún 
más las posibles explicaciones de la sociedad partiendo de la energía.

En el último tercio del siglo XX se publicaron las investigaciones del físico Ilya Prigogine, en cuya obra destaca el interés por los sistemas termodinámicamente abiertos y, dentro de ellos, describe lo que son las estructuras disipativas. Esto no sólo revolucionó la física, sino que permitió, también, que Richard N. Adams incluyera estas ideas en sus trabajos (Adams, 2001).

Adams, yaconlasnuevasperspectivastermodinámicas, pudo plantear una vasta explicación de la sociedad. Desde su óptica pudo esclarecer, por ejemplo, cómo funcionan las relaciones de poder dentro de la sociedad, cómo son las relaciones con el medioambiente y cómo evoluciona la sociedad (Adams, 2001 y 2007). A partir de estas teorías llegó a ser el autor más conspicuo de la energética social hasta el punto de que los seguidores de esta corriente, posteriores a Adams, se han centrado básicamente en aplicar y ampliar sus ideas. Los dos ejemplos más claros son Roberto Varela y Leonardo Tyrtania, que utilizaron los razonamientos de Adams en Morelos y Oaxaca, respectivamente (Varela, 1984; Tyrtania, 2009).

Ahora bien, ¿cómo pueden estas ideas ayudar a encontrar la base física del fenómeno de la alteridad?

\section{El otro como estructura disipativa alejada del equilibrio}

Según la termodinámica, las estructuras disipativas son estructuras autoorganizadas que contienen en sí mismas los elementos para mantenerse durante cierto período de tiempo y que emergen en el proceso de selección natural. También son llamadas estructuras de insumoproducto. Algunos ejemplos de estructuras disipativas son la flama de una vela, la vibración de una cuerda de violín o, incluso, algo tan grande y complejo como una ciudad (Blackburn, 1973: 24; Prigogine, 1977: 53). Ahora bien, todas las estructuras disipativas, sin importar lo complejas que sean, poseen cuatro características que las identifican: dependencia del flujo energético, homeostasis, sucesión y limitación por el costo de transportación de masa (Blackburn 1973:24-26), las cuales describimos a continuación:
1. Dependencia del flujo energético: todo sistema disipativo desaparece si se interrumpe el flujo energético. Esto quiere decir que el otro desaparece como tal si se interrumpen los insumos de energía que lo hacen funcionar.

2. Homeostasis: literalmente, es la autorregulación de la constancia de las propiedades de los sistemas influidos por agentes exteriores. Esto se refiere a que deben estar expuestos a flujos energéticos que tengan la capacidad para desorganizarlos, pero, pese a dichos flujos, prevalecer. Así, el otro está constantemente regulando las entradas y salidas de materia y energía para poder mantenerse.

3. Sucesión: se refiere a que muestra un cambio de propiedades desde su inicio hasta que alcanza su estado "estable". En lo general, el otro se desarrollará desde que nace hasta que llega a la adultez - o clímax-.

4. Limitación por el costo de transportación de masa: las estructuras disipativas alcanzan su estado clímax cuando toda la cantidad posible de materia se ha incorporado a los ciclos materiales. Así, el otro después del clímax seguirá presentando estadios degradados hasta alcanzar el equilibrio termodinámico, esto es, la muerte (Tyrtania, 2009: 79).

Ante esta descripción puede aseverarse que los organismos vivos son estructuras disipativas, sin importar si son vegetales, animales o humanos. Esto está en conformidad con autores como Schneider et al. (1994: 13-16), Blackburn (1997: 24-26) y Margulis (2000: 16). Ahora bien, si todo ser vivo es una estructura disipativa, entonces el otro, al estar vivo, es una estructura disipativa. ¿Qué implicaciones conlleva esto?

\section{Lo que implica concebir al otro como estructura disipativa}

El concebir al otro como estructura disipativa permite comprenderlo de una forma diferente de manera esencial, como primer punto, para entender los flujos energéticos y materiales que le son necesarios para permanecer —alimento, resguardo, excreción-, lo 
cual supone una primera aproximación tanto para el entendimiento de su actividad, como para las posibles motivaciones que el otro tiene para la acción. Además, como segundo punto, facilita comprender el uso que el otro hace del espacio, lo que tiene que ver con los lugares que le son favorables - o siquiera posibles - y aquellos que no lo son. Asimismo, proporciona información sobre su dinámica, su desplazamiento y la apropiación que hace del espacio.

Pero también, como tercer punto, concebir al otro como estructura disipativa permite replantear las posibles relaciones con los otros. Someramente se pueden proponer cuatro tipos principales de relaciones posibles: de apropiación, de asociación, de comensalismo y de pérdida.

1. Relaciones de competencia o apropiación entre las estructuras disipativas: suceden cuando una estructura disipativa "a" y una estructura disipativa "b" compiten entre sí en la búsqueda de ser más eficientes para obtener, o retener, un recurso, sea éste de materia, de energía o de información. Aquí se incluyen todas las relaciones de competencia y depredación.

2. Relaciones de asociación entre las estructuras disipativas: se presentan cuando una estructura disipativa "a" y una estructura disipativa "b" - y hasta una estructura disipativa "n"- se asocian o agrupan para obtener, o retener, un recurso, sea éste de materia, de energía o de información, que también pretende - conservar o adquirir - una estructura disipativa "c". Ha de destacarse que "c" también puede ser una estructura dispativa asociada.

3. Relaciones de comensalismo entre las estructuras disipativas: estas relaciones ocurren cuando una estructura disipativa “a” disipa un recurso, sea éste de materia, de energía o de información, que sólo lo es para la estructura disipativa "b" - para la "a" ya no es un recurso porque no puede utilizarlo en virtud de que lo está disipando-.

4. Relaciones de pérdida: este tipo de relación acontece cuando existe un intento de apropiación, de asociación o de comensalismo por parte de una o más estructuras disipativas, pero esta tentativa no se completa, lo que genera así pérdida en términos de energía, de materia o de información - a uno o a todos los implicados.

Lo anterior es cierto para todas las estructuras disipativas orgánicas, ${ }^{3}$ sean éstas biológicas o sociales, singulares —individuos - o plurales — poblaciones, ciudades, naciones-.

Estas categorías relacionales que se desprenden de la propuesta energética no contravienen a aquellas empleadas en otras disciplinas o teorías. Así, las interacciones ecológicas propuestas por Odum en su libro Ecología: el puente entre ciencia y sociedad no son cuestionadas, ni se desecha la tipología de las relaciones con el otro aceptada por Todorov en su obra La conquista de América: el problemadel otro (Odum, 1998; Todorov, 2001). De igual manera, tampoco se opone a concepciones más recientes, como la denominada de tres figuras de tipos ideales de alteridad, propuesta por Pirni en 2013.

Odum acepta que el efecto que una especie puede tener en otra consigue resumirse en tres opciones (Odum, 1998: 185-189): cuando de alguna manera se beneficia, se dice que la relación es positiva (+); cuando de alguna forma se perjudica, se acepta que la relación es negativa (-); y, además, se admite un tercer caso en el cual la interacción no es ni para bien ni para mal, de ahí que se llame neutra (0). De estas posibilidades, para dos especies, el autor genera un cuadro con trece opciones. ${ }^{4}$ Ninguna de estas posibilidades se elimina desde la perspectiva de la energética, solamente se renombran.

Del mismo modo, en su tipología de las relaciones con el otro, Todorov acepta la existencia de, por lo menos, tres ejes o planos fundamentales que pueden ayudar a situar la problemática de la alteridad. Dichos planos son el axiológico, el praxeológico y el epistémico. Todorov dice textualmente:

El primero, axiológico, se plantea como un juicio de valor: el otro es bueno o malo, lo quiero o no lo quiero, es igual o es inferior a mí. El segundo, praxeológico, me acerca o me aleja en mi relación con el otro; 
adopto sus valores, me identifico con él, o lo asimilo, le impongo mi propia imagen; entre la sumisión al otro y la sumisión del otro hay un tercer punto: la neutralidad o indiferencia. En el tercer plano, el epistémico, conozco o ignoro la identidad del otro, en una gradación infinita en los estados de conocimiento (Todorov, 2001: 195).

Como puede versey, de hecho, lo acepta Todorov mismo, estos tres planos no pueden deducirse uno a partir del otro. Eso no niega que pueden presentar cierta afinidad, pero no se puede establecer, a ciencia cierta, cómo se comportará uno por conocerse el comportamiento del otro. Es decir, no se puede asumir una correspondencia unívoca entre los elementos descriptivos dentro de cada uno de los ejes.

Entonces, la tipología propuesta por Todorov, lejos de refutar la de Odum o invalidarla, la complementa. Esto se evidencia porque, de facto, se propone un cuarto eje - el energético- que resulta ser afín a los mencionados por él, pero que tampoco puede reducirse a los otros ni los otros a él.

La explicación de la alteridad a partir de tres figuras de tipos ideales compagina también con la propuesta energética aquí planteada. En este sentido, Pirni propuso, básicamente, la existencia de tres tipos de alteridad: la alteridad muro, la alteridad espejo y la alteridad puerta (Pirni, 2013).

Según Pirni, la alteridad-muro es la que precede fenomenológicamente a cualquier otra. La razón es que el otro es percibido como un límite, una barrera contra la que chocan y deben retroceder nuestra voluntad y nuestra capacidad de acción (Pirni, 2013: 342). A continuación, Pirni explica lo que es la alteridad-espejo, la cual consiste en que el otro es el que nos reconoce porque, ante todo, somos semejantes a él, y nosotros lo reconocemos inmediatamente por la misma razón. En esta figura, la alteridad se nos muestra como otroyo-mismo del que cabe recibir más confirmaciones que negaciones de lo que soy y lo que querría ser (Pirni, 2013: 343). Por último, Pirni aborda el significado de la alteridad-puerta. En esta figura se alude a la capacidad del otro de volverse medium unificationis de extremos escindidos entre sí, de crear "conexiones" entre diversidades. La alteridad puerta implica, ante todo, la instauración de una dialéctica dentro/fuera, apertura/ clausura (Pirni, 2013: 344).

La revisión de las formas de alteridad de Pirni muestra que sus planteamientos no se oponen a la propuesta del otro desde la energética social. Esto se debe a que la obra de este autor enfoca la superficie, la frontera; es decir, los objetos metaforizados tienen su valor funcional en la superficie, y lo que los identifica a unos y a otros es, precisamente, la capa externa. Así, el muro se distingue del espejo en la incapacidad del primero de reflejar, característica que sí posee el segundo, mientras que los dos primeros se diferencian del tercero en la capacidad del último para abrir y cerrar - detener y dejar pasar- que no poseen los demás. Justamente por centrarse en la frontera, la concordancia con la propuesta de este artículo es posible, ya que las estructuras disipativas tienen fronteras para materia, energía e información que pueden abrir en caso de ser necesario, permitiendo así las interacciones entre estructuras disipativas.

Pero más allá de solamente enlistar relaciones posibles con los otros desde la energética y armonizarlas con otros sistemas de clasificación de relaciones, esta propuesta permite evidenciar grupos de otros que suelen ser ignorados. Con esta perspectiva, los otros no humanos emergen de forma nítida, y tanto el otro vegetal como el otro animal se hacen visibles. Todos aquellos que han sido desprovistos, despojados, desalojados de su otredad (Parrilla-Rubio, 2007:71) la recuperan ya que ellos también son estructuras disipativas orgánicas y, en virtud de eso, pueden ser vistos como otros.

Ahora bien, el trasladar al animal —y al vegetala la esfera de la alteridad también tiene sus propias implicaciones. En primer lugar, que los problemas ecológicos son problemas sociales; en segundolugar, que espacialmente no existe una razón para la segregación; en tercer lugar, que las estrategias de conservación deben realinearse con este hecho; y, finalmente, que si se desea entender a detalle el funcionamiento de los asentamientos humanos, se debe considerar al otro no humano también. 
Los problemas ecológicos son, originalmente, problemas sociales. Este razonamiento ha sido planteado de diversas maneras por varios autores como Adams (2001: 47), Miranda-Vega (1997: 73) y JullianMontañez (2013: 146), entre otros. La lógica de fondo para estas posturas es la misma, que las principales fuentes de estrés - y daño- para los ecosistemas provienen de la actividad humana, de modo que, si se modera la acción humana, se moderarán los problemas ecológicos. De manera concisa, pueden retomarse las palabras de Jullian-Montañez: "puede afirmarse que la conservación de especies animales es un asunto de alteridad. Si bien la componente biológica [sic] es innegable, en el fondo, se trata sólo de permitirle vivir al otro, al que es distinto" (Jullian-Montañez, 2013: 146). Estas palabras concuerdan con el sentido de otras propuestas derivadas de la filosofía. Concretamente, se asemejan a la igualdad biosférica -igualdad de derecho a vivir y florecer - y al principio ecológico de vivir y dejar vivir, que se relacionan con la denominada ecología profunda (Naes, 2007). También guardan relación con la necesidad de llevar a las esferas práctica y ético-política el cambio de actitud, retomando las propuestas de las tres ecologías (Guattari, 1996).

Además, espacialmente, no existe una razón para la segregación del otro no humano, incluso en los asentamientos humanos. Es decir, al ser estructuras disipativas orgánicas no humanas, tanto la fauna como la flora están y seguirán estando presentes en las ciudades. La razón es que ahí pueden cubrir sus necesidades de intercambio - de materia, energía e información - con el medio y, por eso, permanecen. En la literatura científica abundan las investigaciones biológicas que dan cuenta del fenómeno de la fauna que reside en las ciudades - por citar sólo unos ejemplos, Oprea et al. (2009), Nemeth (2010), Threlfall et al. (2012), Bonier (2012) y López-Berrizbeitia (2013) - .

Las estrategias de conservación de flora y fauna actuales se han elaborado asumiendo la separación humano-naturaleza. Es decir, giran en torno a áreas de exclusión -áreas naturales protegidas y herramientas semejantes - que no atacan el problema de fondo ya que no promueven la coexistencia humano-naturaleza. Por lo tanto, parece pertinente reajustarlas ${ }^{5}$ o realinearlas tomando en consideración las relaciones energéticas de las estructuras disipativas involucradas. Por ejemplo, Jullian-Montañez (2013) propuso cuatro áreas o dominios de oportunidad donde se podía actuar para favorecer la coexistencia humano-naturaleza — dichos dominios eran los siguientes: educación ambiental, monitoreo, infraestructura/servicios y vinculación-.

También, para entender a detalle el funcionamiento de los asentamientos humanos — sobre todo de la ciudad-, se debe considerar al otro no humano porque, aunque este componente ha sido ignorado, su presencia es abundante y su actividad muy significativa en dichos lugares. Así, la vegetación contribuye a la remoción de $\mathrm{CO}_{2}$ de la atmósfera (Ramírez et al., 2012), a mejorar la calidad del ambiente (Malea, 2013), como termorregulador (Frumkin, 2002; Llanque-Chana, 2003) y, según Alcalá et al. (2008), incluso puede servir como indicador para evaluar la contaminación por metales pesados. Del mismo modo, la fauna presente en la ciudad cubre varias funciones, como el control de plagas, la polinización o como bioindicadores (Cleveland et al., 2006; Cabidoet al., 2008). Por supuesto, también presentan riesgo de zoonosis (Canteros et al., 2005). Por lo anterior, si se desea dar cuenta del funcionamiento de la ciudad -como aquellas investigaciones propuestas por el metabolismo social_- ${ }^{6}$ éste es un elemento que no puede ignorarse (Villegas et al., 2008; Jullian-Montañez, 2013).

En este sentido, volviendo a la pregunta que planteó González-Silva (2009b), “iserá la alteridad el eslabón perdido que permita el re-encuentro verdadero de los habitantes en esta aldea global?", se puede responder que es posible que desde la base material de la alteridad se facilite la identificación del otro y se puedan reconocer sus necesidades, su espacialidad y sus capacidades de relación. Por otra parte, aceptar que la alteridad pueda hacer mucho más partiendo solamente del enfoque energético resulta pretencioso.

\section{A manera de conclusión}

Al margen de las ideas, creencias, formaciones y prejuicios que se tengan, los seres vivos están definidos, 
desde el punto de vista de la física, como estructuras disipativas, sin importar si se es vegetal, animal o humano. Es pertinente destacar que cuando se afirma esto no se hace de manera metafórica. Parafraseando a Adams, "la segunda ley de la termodinámica — de donde surge la noción de estructura disipativa - es atractiva para los científicos sociales porque puede aplicarse directamente a los fenómenos sociales" (Adams, 2007: 73-74); es decir, que en última instancia la forma más simple de definir al otro desde el punto de vista de su existencia material es afirmar que el otro es una estructura disipativa orgánica, lo que obliga a voltear la vista hacia el significado y las implicaciones que esto tiene. Por otra parte, el no hacerlo implica ignorar la base física del fenómeno y, por tanto, caer en el equívoco de asignarle propiedades o límites improbables, o de plano físicamente imposibles.

Por supuesto, la termodinámica impone límites, pero hay que tener cuidado de no reducir toda la explicación del fenómeno a ella. Al respecto, Georgescu-Roegen advirtió: "La termodinámica impone un límite a estos fenómenos, pero no los gobierna" (Georgescu-Roegen, 1981: 129). Es decir, los fenómenos biológicos y sociales están supeditados a la termodinámica, pero adoptan sus propias dinámicas debido al nivel de complejidad que poseen, de ahí que cada uno de esos niveles posea comportamientos que le son propios y distintivos. Es, para decirlo en palabras de Leonardo Tyrtania, de esta manera: "En cada nivel aparecen potencialidades inéditas y nuevos códigos de procesamiento de información" (Tyrtania, 2009: 55).

Por tanto, se puede concluir que los límites de la alteridad, desde el punto de vista material $-y$ energético-, están cimentados en la termodinámica de los sistemas del no equilibrio. Los otros límites - los biológicos, sociales, filosóficos, pedagógicos y otroshabrá que buscarlos en las relaciones y constricciones que dichos límites físicos permitan.

\section{Notas}

${ }^{1}$ Asumiendo, claro está, los seres aceptados por la ciencia actual que poseen un cuerpo físico. Para aquellos seres descritos por la religión, que carecen del componente material, es posible que la propuesta de este trabajo no cuadre.

${ }^{2}$ Sobre este particular resulta muy interesante la obra de Levinas, que llega a hablar incluso de la huella del otro; es decir, de la impronta en el tiempo del otro. Sin embargo, también Levinas deja de lado los elementos físicos del otro y termina por asignar la alteridad al género femenino.

${ }^{3}$ Se entiende aquí por estructura disipativa orgánica toda aquella que está compuesta por biomoléculas - proteínas, lípidos, carbohidratos y ácidos nucléicos-y que presenta algún tipo de metabolismo.

${ }^{4}$ Las relaciones que menciona Odum son las siguientes: mutualismo $(++)$, protocooperación $(++)$, comensalismo $(+0)$, inquilinismo $(+0)$, parasitismo $(+-)$, depredación $(+-)$, herbivorismo (+-), amensalismo (00), apatía o neutralismo (00), antibiosis (0-), alelopatía (0-), competencia (-) y antagonismo, también llamado negativismo (-) .

${ }^{5}$ Conviene hacer una acotación sobre esto. Existe fauna que, por su peligrosidad para el humano, no puede ser considerada para coexistir en los asentamientos humanos. Solamente para este tipo de fauna y para aquella que no tolera vivir en compañía del humano puede resultar viable su conservación a través de las áreas naturales protegidas. Pero aún estas especies se verían beneficiadas si los planes de conservación se centraran en las relaciones -entre ellas y con el humano-y no únicamente por su ubicación geográfica o por la presencia de alguna especie carismática o seriamente amenazada.

${ }^{6}$ Toledo expresó que queda pendiente el análisis del metabolismo social de la ciudad (Toledo, 2008). Es por eso que se plantea como necesario incluir en dicho metabolismo la actividad del otro no humano; es decir, las plantas y los animales.

\section{Referencias bibliográficas}

Adams, Richard Niebold (2001), El octavo día. La evolución social como autoorganización de la energía. México, UAM. 
Adams, Richard Niebold (2007), La red de la expansión humana. México, UAM.

Alcalá, J. et al. (2008), "Metales pesados en vegetación arbórea como indicador de la calidad ambiental urbana: ciudad de Chihuahua, México". En Multequina, vol. 17, pp. 39-54.

Baudrillard, Jean (1997), El otro por sí mismo. España, Anagrama.

Blackburn, Thomas R. (1973), "Information and the Ecology of Scholars". En Tyrtania, Leonardo, Termodinámica de la supervivencia para las ciencias sociales. México, UAM, pp. 21-42

Bonier, F. (2012), "Hormones in the city: Endocrine Ecology of Urban Birds". En Hormones and Behavior, vol. 61, pp. 763-772.

Cabido, Carlos, Adega Gonzalo, Pilar López y José Martín (2008), Poblaciones urbanas de la lagartija ibérica: uso como bioindicador de los efectos del ambiente urbano. XIX Premio de Medio Ambiente. España, Caja de Ahorros y Monte de Piedad de Segovia.

Canteros, C.E. et al. (2005), "Primer aislamiento de Histoplasma capsulatum de murciélago urbano Eumops bonariensis". En Revista Argentina de Microbiología, vol. 37, pp 46-56.

Cleveland, C.J. et al. (2006), "Economic Value of the Pest Control Service Provided by Brazilian FreeTailed Bats in South-Central Texas". En Ecological Environment, vol. 4, núm. 5, pp. 238-243.

Frumkin, H. (2002), “Urban Sprawl and Public Health". En Public Healt Reports, vol. 117, pp. 201- 217.

Georgescu-Roegen, Nicholas (1981), “Afterword". En Tyrtania, Leonardo, Termodinámica de la supervivencia para las ciencias sociales. México, UAM, pp. 119-130.

González-Silva, Freddy (2009a), "Alteridad y su itinerario desde las perspectivas multidisciplinares". En Revista Reflexiones, vol.88, núm. 1, pp. 119-135.

González-Silva, Freddy (2009b), "Itinerario de alteridad. Una reconstrucción para nuevas aproximaciones". En Fermentum, núm. 56, pp. 616-637.

Guattari, Félix (1996), Las tresecologías. España, Pre-textos. Jullian-Montañez, Alain Gabriel (2013), Fauna silvestre en las ciudades. Estudio de caso: Quiropterofauna en Ensenada,
Baja California, México. Tesis doctoral. Universidad Autónoma de Baja California, México.

Llanque-Chana, J. (2003), "Efectos de la contaminación atmosférica en el clima urbano y calidad ambiental de Arequipa". En Cuadernos de Investigación Urbanística, núm. 37, pp. 95-106.

López Berrizbeitia, M.F. y M.M. Díaz (2013), "Diversidad de murciélagos (mammalia, chiroptera) en la ciudad de Lules, Tucumán”. En Acta Zoológica Mexicana, vol. 29, núm. 1, pp. 234-239.

Malea, N.C. (2013), "Contribución de las áreas verdes urbanas a la calidad ambiental del distrito de ComasLima, al año 201l". En Revista del Instituto de Investigación (RIIGEO), FIGMMG-UNMSM, vol. 15, núm. 30, pp. 117-121.

Margulis Lynn, Dorion Sagan (2000), What is life? Canada, Simon \& Shuster.

Miranda-Vega, Clara Elisa (1997), Filosofíaymedio ambiente, una aproximación teórica. México, Taller Abierto.

Naes, A. (2007), "Los movimientos de la ecología superficial y la ecología profunda: un resumen”. En Revista Ambiente y Desarrollo, vol. 23, núm. 1, pp. 98-101. Nemeth, E. y H. Brumm (2010), "Birds and Antropogenic Noise: Are Urban Songs Sdaptative?" En The American Naturalist, vol. 176, núm. 4, pp. 465-475.

Odum, Eugene P. y Fausto O. Sarmiento (1998), Ecología: el puente entre ciencia y sociedad. México, Mc Graw HillInteramericana.

Oprea M., P. Mendes y T.B. Vieira (2009), “Do Wooded Streets Provide Connectivity for Bats in an Urban Landscape?" En Biodiversity Conservation, núm. 18, pp. 2361-2371.

Parrilla-Rubio, María Victoria (2007), "Tan otro que ni otredad posee: eso que llaman el animal”. En Thémata Revista de Filosofía, núm. 39, pp. 71-78.

Pirni, A. (2013), “¿Contra Schmitt? Modelos de alteridad para la convivencia”. En Bajo Palabra, Revista de Filosofía. II Época, núm. 8, pp. 335-352.

Prigogine, Ilya (1977), "Tendencias a largo plazo y evolución de la complejidad". En Tyrtania, Leonardo, Termodinámica de la supervivencia para las ciencias sociales. México, UAM, pp. 43-118.

Ramírez, W.A. y T. Bolaños-Silva (2012), "Revisión sobre el papel de los techos verdes en la remoción 
de carbono atmosférico en el neotrópico". En Nodo, vol. 6, núm. 12, pp. 7-18.

Ricoeur, Paul (1996), Sí mismo como otro. México, Siglo XXI.

Schneider, E.D.yJ.J. Kay (1994), “Life as a Manifestation of the Second Law of Thermodynamics". En Mathematical and Computer Modelling, vol 19, núm. 6-8, pp. 25-48.

Skliar, Carlos (2002), iY si el otro no estuviera ahí? Notas para una pedagogia (improbable) de la diferencia. Argentina, Miño y Dávila.

Threlfall C.G., B. Law y P.B. Banks (2012), "Sensitivity of Insectivorous Bats to Urbanization: Implications for Suburban Conservation Planning". En Biological Conservation, núm. 146, pp. 41-52.

Todorov, Tzvetan (2001), La conquista de América. El problema del otro. México, Siglo XXI.

Toledo, Víctor Manuel (2008), "Metabolismos rurales: hacia una teoría económico-ecológica de la apropiación de la naturaleza". En Revista Iberoamericana de Economía Ecológica, vol. 7, pp. 1-26.

Tyrtania, Leonardo (2008), "La indeterminación entrópica. Notas sobre disipación de energía, evolución y complejidad". En Desacatos. Revista de Antropología Social, núm. 28, pp. 41-68.

Tyrtania Leonardo (2009), Evolución y sociedad. Termodinámica de la supervivencia para una sociedad a escala humana, México, UAM-Juan Pablos.

Varela Roberto (1984), Expansiónde sistemas y relaciones de poder: antropología política del estado de Morelos. México, UAM.

Villegas, M.B. y A. Garitano-Zavala (2008), "Las comunidades de aves como indicadores ecológicos para programas de monitoreo ambiental en la ciudad de La Paz, Bolivia”. En Ecología en Bolivia, vol. 43, núm. 2, pp. 146-153.

White, Leslie (1943), "Energy and the Evolution of Culture". En American Anthropologist New Series, vol. 45, núm. 3, pp. 335-356. 